\title{
Inhibition of CXCR4 in Spinal Cord and DRG with AMD3I00 Attenuates Colon-Bladder Cross-Organ Sensitization
}

\author{
Hengshuai Zhang (ID) \\ Xingyou Dong \\ Zhenxing Yang \\ Jiang Zhao \\ Qudong Lu \\ Jingzhen Zhu \\ Longkun $\mathrm{Li}$ \\ Shanhong $\mathrm{Yi}$ \\ Jie $\mathrm{Xu}$ (D)
}

Department of Urology, Second Affiliated Hospital, Army Medical University,

Chongqing, 400037, People's Republic of China
Correspondence: Jie $\mathrm{Xu}$; Shanhong Yi Department of Urology, Second Affiliated Hospital, Army Medical University, Chongqing, 400037, People's Republic of China

Email xujiel98I@tmmu.edu.cn; 2644057844@qq.com
Background: Cross-sensitization of pelvic organs is one theory for why symptoms of gut sickness and interstitial cystitis/bladder pain syndrome overlap. Experimental colitis has been shown to trigger bladder hyperactivity and hyperalgesia in rats. The chemokine receptor CXCR4 plays a key role in bladder function and central sensitization. We aim to study the role of CXCR4 and its inhibitor AMD3100 in colon-bladder cross-organ sensitization.

Methods: The colitis model was established by rectal infusion of trinitrobenzene sulfonic acid. Western blot and immunofluorescence were used to assess the expression and distribution of CXCR4. Intrathecal injection of AMD3100 (a CXCR4 inhibitor) and PD98059 (an ERK inhibitor) were used to inhibit CXCR4 and downstream extracellular signal-regulated kinase (ERK) in the spinal cord and dorsal root ganglion (DRG). Intravesical perfusion of resiniferatoxin was performed to measure the pain behavior counts of rats, and continuous cystometry was performed to evaluate bladder voiding function.

Results: Compared to the control group, CXCR4 was expressed more in bladder mucosa and colon mucosa, L6-S1 dorsal root ganglion (DRG), and the corresponding segment of the spinal dorsal horn (SDH) in rats with colitis. Moreover, intrathecal injection of the AMD3100 suppressed bladder overactivity, bladder hyperalgesia, and mastocytosis symptoms caused by colitis. Furthermore, AMD3100 effectively inhibited ERK activation in the spinal cord induced by experimental colitis. Finally, treatment with PD98059 alleviated bladder overactivity and hyperalgesia caused by colitis.

Conclusion: Increased CXCR4 in the DRG and SDH contributes to colon inflammationinduced bladder overactivity and hyperalgesia partly via the phosphorylation of spinal ERK. Treatment targeting the CXCR4/ERK pathway might provide a potential new approach for the comorbidity between the digestive system and the urinary system.

Keywords: pain, visceral hypersensitivity, sensory afferents, urgency, colitis, ERK

\section{Introduction}

Interstitial cystitis/bladder pain syndrome (IC/BPS) is an etiologically unclear chronic pain condition characterized by bladder-related pain and discomfort, troubled frequent urination, and nocturia. ${ }^{1}$ Growing evidence shows that chronic bladder dysfunction represented by IC/BPS is closely related to gut diseases. ${ }^{2,3}$ Gut diseases are ten to one hundred times more frequent in IC/BPS patients compared to the general population. ${ }^{4}$ Similarly, as reported in a recent long-term cohort study, patients diagnosed with irritable bowel syndrome (IBS) are more likely to have IC/BPS. ${ }^{5}$ 
The "cross-organ sensitization" hypothesis is currently a widely accepted hypothesis to describe and explain the common pathogenesis of abnormal urination diseases and intestinal diseases. ${ }^{2}$ The part of neurons in DRG and spinal cord that can receive afferents from the bladder and colon at the same time is the anatomical basis of this hypothesis, and peripheral and central sensitization are the key events. ${ }^{6,7}$ In rats with colitis, the excitability of sensory afferent fibers and corresponding dorsal root ganglion (DRG) neurons in the bladder increased significantly, and the bladder was more sensitive to chemical stimuli such as capsaicin, carbachol, and bradykinin. ${ }^{89}$ At the spinal cord level, evidence suggests that the activation of microglia promotes bladder hypersensitivity caused by colitis. ${ }^{10}$ These studies indicate the important role of central and peripheral sensitization in bladder hypersensitivity after colitis. However, the mechanism leading to sensitization of the common neural pathways of the bladder and colon remains unclear.

CXCR4 is a key receptor for the chemokine CXCL12 and is widely involved in inflammation and neuroregulation. Evidence shows that CXCR4 participates in the induction of central and peripheral sensitization. ${ }^{11}$ It is widely distributed in neurons and glial cells of DRG and spinal cord, and intrathecal injection of CXCL12 to activate CXCR4 contributes to mechanical hyperalgesia. ${ }^{12}$ CXCR4 knockdown results in significantly higher nociceptive thresholds than wild-type rats in response to intraplantar complete Freund's adjuvant injection. ${ }^{13}$ In addition, AMD3100 prevents bladder hyperalgesia induced by PAR activating peptides. ${ }^{14}$ The significant increase in voiding interval caused by the CXCR4 receptor antagonist AMD3100 indicates that the CXCR4 receptor has a promoting effect on the voiding reflex. ${ }^{15}$ Together, these findings suggest that CXCR4 is critical for central and peripheral sensitization and is involved in overactive bladder and bladder hyperalgesia. A previous study found that the number of bladder sensory-related neurons sensitive to CXCR4 signals in the DRG significantly increased after sciatic nerve injury, ${ }^{16}$ suggesting a possible link between CXCR4 and mutual interference involving different diseases. However, it is less clear whether CXCR4 contributes to colon-bladder cross-organ sensitization.

Thus, in the present study, we established a trinitrobenzene sulfonic acid (TNBS)-induced chronic colitis model in rats and investigated whether CXCR4 is altered in the lumbosacral spinal cord and corresponding ganglia. Additionally, we examined the impact of
AMD3100 on bladder hyperactivity and hyperalgesia, and whether ERK signals in the lumbosacral spinal cord and corresponding ganglia are involved as possible downstream.

\section{Materials and Methods}

\section{Animal}

Female Sprague Dawley rats $(200-250 \mathrm{~g})$ were obtained from the Laboratory Animal Center of the Army Medical University. Animals were raised between $22-24^{\circ} \mathrm{C}$ and given unrestricted access to food and water. All animal tests were conducted according to the Guidelines for the Care and Use of Laboratory Animals of the Chinese Animal Welfare Committee and approved by the Animal Care and Use Committee of the Army Military Medical University (approval No. AMUWEC20212093).

\section{Induction of Experimental Colitis by TNBS}

As previously described, ${ }^{17}$ isoflurane was used to anesthetize the rats following a 24-hour fast. Next, a PE-50 catheter was introduced into the colon from the anus and the end was $8 \mathrm{~cm}$ deep. Then an equal volume of mixed TNBS solution and absolute ethanol was injected into the colon at a final concentration of $80 \mathrm{mg} / \mathrm{kg}$. The rats were then positioned supine with the lower body elevated to $60^{\circ}$ to reduce drug leakage. Correspondingly, the same volume of $50 \%$ ethanol was administered to the control (CON) group.

\section{Drugs and Intrathecal Injection}

Intrathecal administration was similar to those reported before. ${ }^{18}$ CXCR4 inhibitor AMD3100 and ERK inhibitor PD98059 were purchased from MCE (MCE, China). Following intraperitoneal anesthesia of rats with $100 \mathrm{mg} /$ $\mathrm{kg}$ pentobarbital, the skin at the level of the fourth through fifth lumbar vertebrae on the back of the mouse was incised. A partial laminectomy at L4/L5 was performed to expose the dura and a 30-gauge needle was placed in the midline into the $L 4 / 5$ intervertebral region. A catheter with an external diameter of $0.2 \mathrm{~mm}$ was inserted into the needle hole, and its end was pushed into and terminated at the L6/S1 vertebral level. Subsequently, a 6-0 silk thread was used to secure the catheter's insertion end to the surrounding tissue. To facilitate administration, the free end of the catheter is sent subcutaneously to the back of the neck to be exposed and fixed. After the catheter 
implantation, all rats recovered for 2 days prior to experiments. AMD3100 (10ug/20ul), PD98059 (5ug/20ul), or an equivalent amount of solvent were delivered to rats through the intrathecal catheter for 7 consecutive days in separate groups.

\section{Tissue Sections and Staining}

One week after the colitis model was established, the rats were slaughtered and tissues including the bladder, distal colon, DRG, and spinal cord were taken. A part of these tissues used for tissue staining was fixed in 4\% paraformaldehyde and embedded in paraffin. The fixed bladder, colon, and spinal cord tissues were cut into $5 \mu \mathrm{m}$ thick sections, and DRG tissue was cut into $20 \mu \mathrm{m}$ thick sections. The histomorphological changes of the colon and bladder were analyzed by hematoxylin-eosin (HE) staining and mast cell enumeration was performed by toluidine blue staining. The above staining was carried out following the standard procedures.

\section{MPO Activity Measurement in the Bladder and Colon}

The activity of myeloperoxidase (MPO) in bladder and colon tissues was measured using a kit purchased from Nanjing Jiancheng Institute of Bioengineering. In brief, the harvested bladder and colon were homogenized with the MPO assay buffer and centrifuged at $12000 \mathrm{~g}$ for 5 minutes, and then the supernatant was used to determine MPO activity according to the kit instructions.

\section{Immunohistochemistry}

Immunohistochemistry was performed as previously described. ${ }^{19}$ The rat bladder section was deparaffinized in a gradient concentration of ethanol solution, and then heated for antigen retrieval. At room temperature, 3\% hydrogen peroxide was used to block endogenous peroxidase. After blocking with 5\% BSA for 1 hour, the sections were incubated with 1:100 PBS containing anticytokeratin 20 antibody (Abcam, UK) and kept at $4{ }^{\circ} \mathrm{C}$ overnight. On the second day, the sections were incubated with HRP-labeled anti-rabbit secondary antibody. After washing the section again, $\mathrm{DAB}$ chromogen was used to mark target proteins, and the nucleus was counterstained with haematoxylin.

\section{Immunofluorescence Staining}

The tissue sections were washed in PBS and blocked with $5 \%$ BSA in PBS for 100 minutes. The sections were washed three times and incubated with the primary antibody CXCR4 (Abcam, UK) or p-ERK1/2 (Abcam, UK) at $4^{\circ} \mathrm{C}$ for 12 hours. Then the sections were washed again in PBS and treated with the secondary antibody diluted in PBS for 100 minutes at room temperature. Goat antimouse Alexa Fluor 555 secondary antibody (Thermo Fisher, USA) was used at 1:1000 dilution. After that, the sections were rinsed with PBS and counterstained with DAPI, then analyzed using an inverted fluorescence microscope (Olympus, Denmark).

\section{Western Blot}

RIPA buffer containing proteinase inhibitors (Beyotime, China) was used to create tissue extracts, which were then used to perform the analyses., and the concentration of protein was measured by BCA Protein Assay Reagent (Beyotime, China). The tissue lysate containing $40 \mu \mathrm{g}$ of total protein was loaded onto the SDS-PAGE gel and electrophoresed at $120 \mathrm{~V}$ for 90 minutes, and then the protein to be tested in the gel was transferred to a $0.45 \mathrm{~m}$ polyvinylidene fluoride membrane (Merck Millipore, Germany). After blocking the membrane in TBST buffer containing 5\% skimmed milk powder for 30 minutes, the membranes were incubated for 12 hours at $4{ }^{\circ} \mathrm{C}$ with the following antibodies: CXCR4 (Abcam, UK), GAPDH (Proteintech, China), $\beta$-tubulin (Proteintech, China), ERK1/2 (Abcam, UK), p-ERK1/2 (Abcam, UK). The membranes were rinsed three times with TBST (5 minutes/wash) the next day before being incubated with goat anti-rabbit secondary antibody (Thermo Fisher, USA) or goat anti-mouse secondary antibody (Thermo Fisher, USA) in 5\% skimmed milk powder /TBST, and then rinsed 3 times. Membrane development was carried out using ECL Substrate (Thermo Fisher, USA), and the chemiluminescence was captured using the bioanalytical imaging system C300 (Azure Biosystems, USA) and analyzed using ImageJ software.

\section{Continuous Cystometry}

Cystometry was performed on unconscious rats 7 days after induction of experimental colitis to evaluate the urodynamic contraction pressure and time as previously described. ${ }^{18}$ Briefly, rat bladders were exposed through a $2 \mathrm{~cm}$ suprapubic incision after they were anesthetized with urethane. Then, through a minimally invasive incision on the top of the bladder, a PE-50 catheter was connected to the bladder lumen and the connection was fixed with surgical sutures. At the end of the operation, the 
PE-50 tube was attached via a three-way connector to a data acquisition system (Chengdu Instrument Factory, China) for recording voiding frequency and pressure, and to a microperfusion pump (Smiths Medical, USA) for infusing saline $(10 \mathrm{~mL} / \mathrm{h}$, room temperature). After $20-$ 30 minutes of continuous perfusion, at least 8 stable urination waveforms for each animal were observed and recorded. The maximum bladder pressure (MBP) and inter contractile interval (ICI) were measured and compared between different groups.

\section{Nociceptive Behavior Observation}

Assessment of bladder nociceptive behavior was performed as described previously, ${ }^{20}$ with slight modification. Rats were adapted to the experimental environment 7 days after receiving TNBS or vehicle treatment, and they were placed in a clear plastic box for at least 2 hours before testing. Thereafter, rats were restrained on the rat fixator and urethral catheterization was performed. After confirming that the bladder is empty, resiniferatoxin (RTX, $0.3 \mu \mathrm{M}, 0.3 \mathrm{~mL}$, dissolved in $80 \%$ physiological saline, $10 \%$ ethanol, and $10 \%$ Tween 80 , Cfwlabs, USA) was then instilled into the bladder through the PE-10 tube. The transurethral catheter remained in place for $1 \mathrm{~min}$ before removal. Following this, the rats were placed back in the plastic box and their behavior was recorded. During this time, an observer counted the number of harmful behaviors. The number of licking behaviors of licking the lower abdomen and freezing behavior of turning the head to the abdomen was recorded. According to 5 seconds as a time unit, the number of time units in which the above behavior occurs in 15 consecutive minutes was recorded separately.

\section{Statistical Analysis}

Statistical analysis was performed using GraphPad Prism 8.0. Results were expressed as median (min-max). Pairwise comparisons were performed using a nonparametric Mann-Whitney test. A Kruskal-Wallis test was used in comparisons between four groups and Dunn's post hoc test was used following the KruskalWallis test. $\mathrm{P}<0.05$ was indicated statistically significant (NS: not significant, ${ }^{*} \mathrm{P}<0.05, * * \mathrm{P}<0.01$, ***P $<0.001$ ).

\section{Results}

\section{Histopathological Features of the Colon} and Bladder

Compared with the CON group, rats after one week of TNBS administration had hypomotility, debilitation and their body weight decreased. The colon of the TNBS group presented with ulceration, mucosal erosion, interstitial congestion, and focal infiltration of inflammatory cells. However, the colon tissues of the control group showed complete epithelial surfaces. The structure of bladder tissues in both groups was intact. The overall structure of the bladder tissue in both groups appeared normal, and the cells were tight and tidy without inflammatory cell infiltration (Figure 1A). TNBS significantly increased the destructive inflammation and tissue damage of the colon but not the bladder as measured by histological score and tissue myeloperoxidase (MPO) levels (Figure 1B and C). The umbrella cell layer is the cell layer in the urothelium that is in direct contact with urine. ${ }^{21}$ We labelled the umbrella cell with cytokeratin 20 (CK20) by immunohistochemistry and found that the umbrella cell layer in the TNBS group was continuous and complete, which was comparable to the CON group (Figure 1D).

\section{Colon Inflammation Increases CXCR4 Expression in the Distal Colon and Bladder}

Immunofluorescence and Western blotting techniques were used to examine the differences in the expression of CXCR4 in the distal colon and bladder under normal and colitis conditions. By immunofluorescence in colon sections from CON and TNBS rats, we observed enhanced immunoreactivity of CXCR4 in the mucous layer from TNBS compared to CON rats (Figure $2 \mathrm{~A}$ and $\mathrm{C}$ ). Mucosal CXCR4 immunoreactivity was also increased in the bladder after colonic inflammation (Figure 2B and D). The Western blot results indicate that colonic inflammation upregulated CXCR4 expression in the colon and bladder by 1.90 -fold and 1.89 -fold, respectively (Figure $2 \mathrm{E}-\mathrm{H}$ ).

\section{TNBS Induces CXCR4 Upregulation in the Dorsal Root Ganglion and Spinal Cord}

Due to the neural crosstalk at the level of DRG and spinal cord is the pathophysiological basis of colon-tobladder crosstalk, we investigated whether experimental colitis upregulates the expression of CXCR4 in the common afferent pathway of the bladder and colon. Immunofluorescence results showed that DRG sections from TNBS treated rats had enhanced immunoreactivity of CXCR4, and a higher number of CXCR4 positive neurons as compared to $\mathrm{CON}$ rats (Figure $3 \mathrm{~A}$ and $\mathrm{B}$ ). 
A
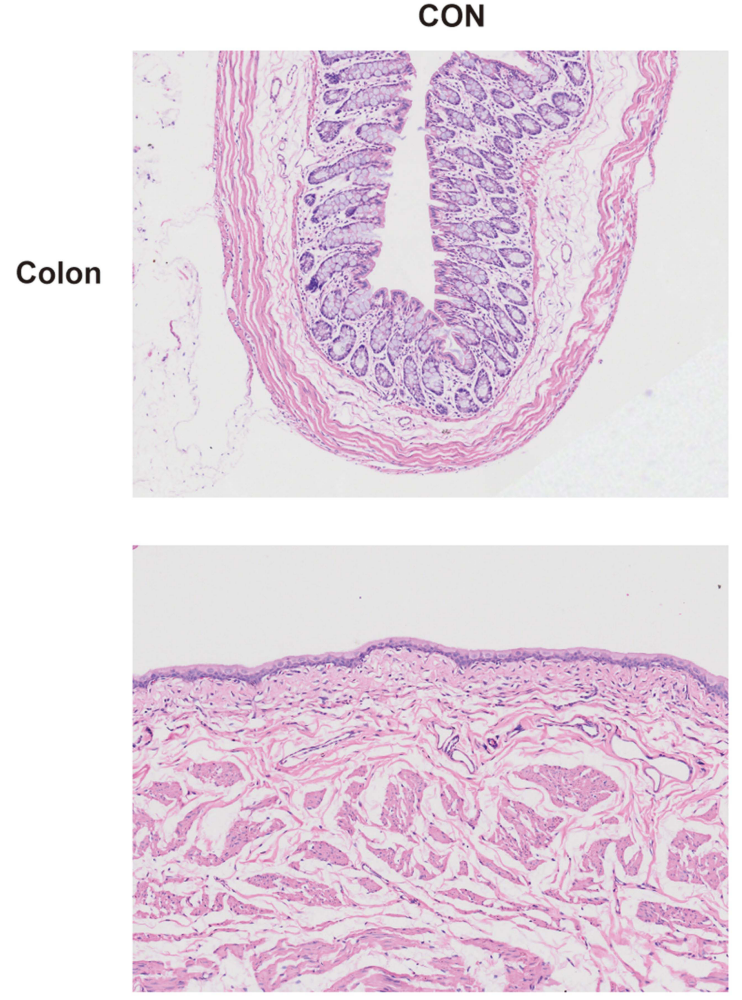

TNBS


C

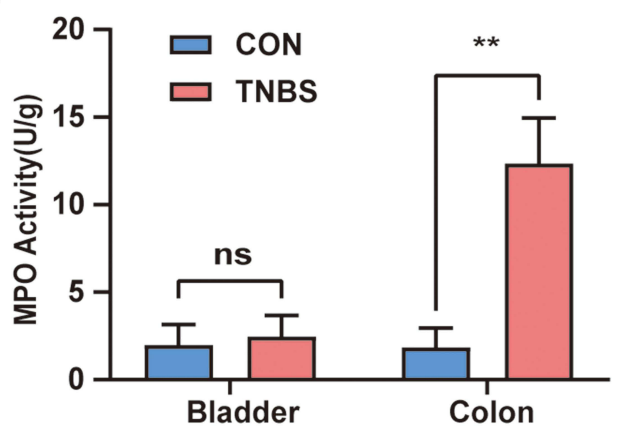

D

B

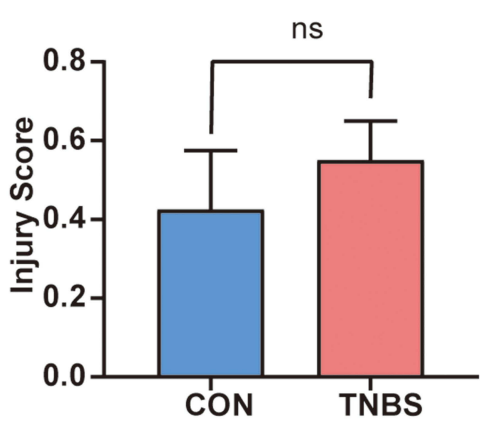

Colon

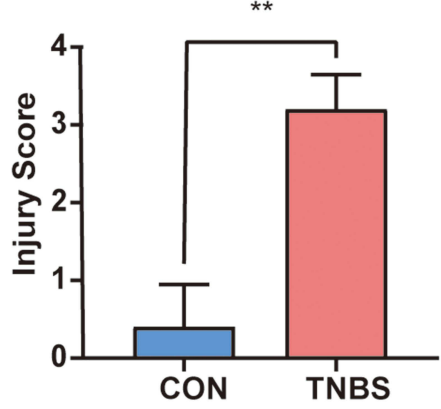

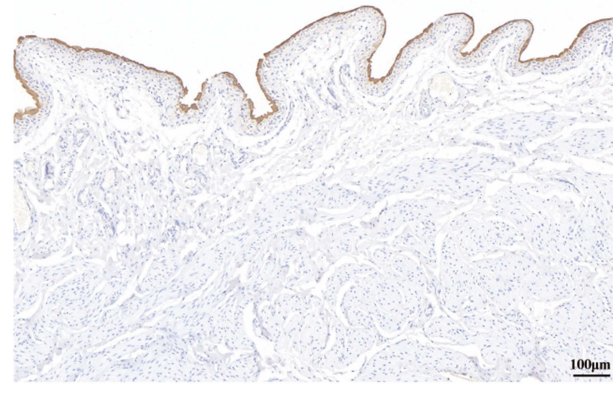

TNBS

Figure I Changes in histology and MPO activity in the colon and bladder during experimental colitis. (A) Colon and bladder tissues are stained with HE. (B) Injury score for colon and bladder tissues $(n=5)$. (C) Units of MPO activity per gram of the colon and bladder $(n=5)$. (D) CK20 immunohistochemistry in the bladder. **P< 0.0 I. Abbreviation: NS, not significant. 
A
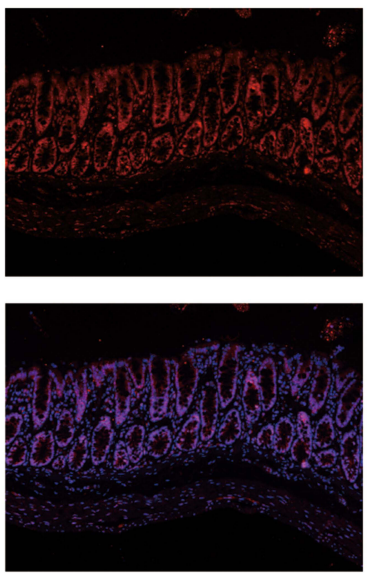

CON
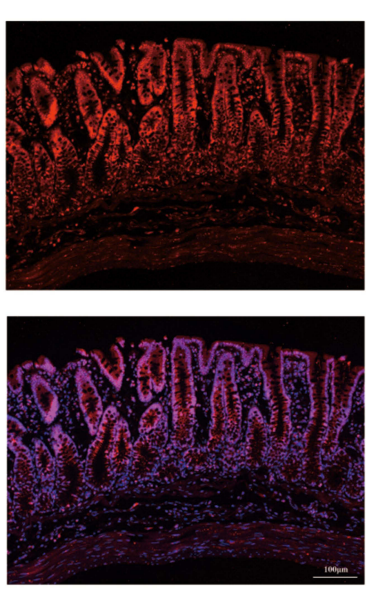

TNBS

\section{B}
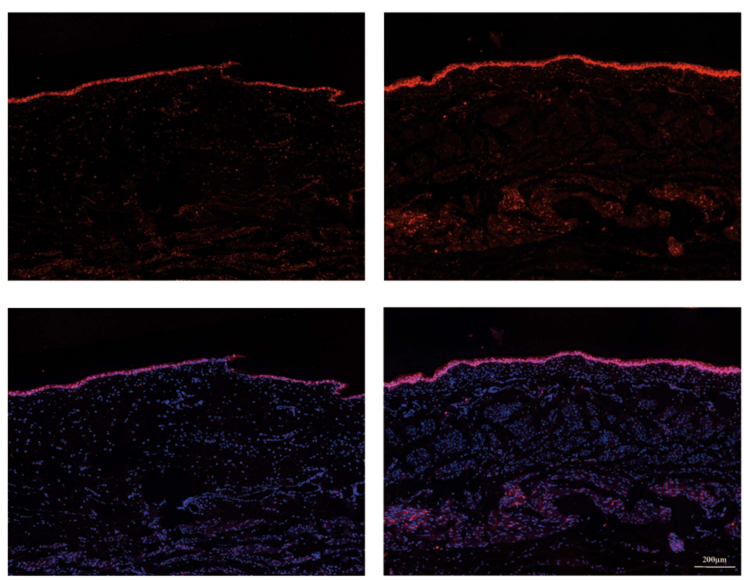

CON

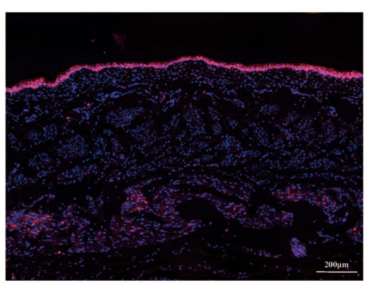

TNBS

D



G

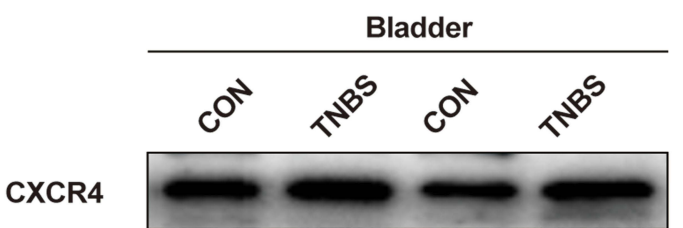

GAPDH

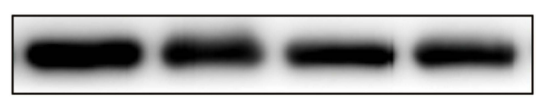

H



Figure 2 The expression of CXCR4 was increased in the colon and bladder after TNBS-induced colitis. (A) Immunofluorescence staining of CXCR4 in the colon and fusion picture of DAPI and CXCR4. (B) Immunofluorescence staining bladder sections, CXCR4 (red), DAPI (blue) are shown. Quantification of the fluorescence intensity in the colon (C) and bladder (D) tissue $(n=5)$. (E) Western blot results of CXCR4 in the colon and (F) quantitative analysis of Western blot analysis normalized to GAPDH $(n=6)$. $(\mathbf{G})$ Western blot results of CXCR4 in the bladder and $(\mathbf{H})$ statistical analysis of Western blotting results $(n=6)$. $* * P<0.0$ I. 
A
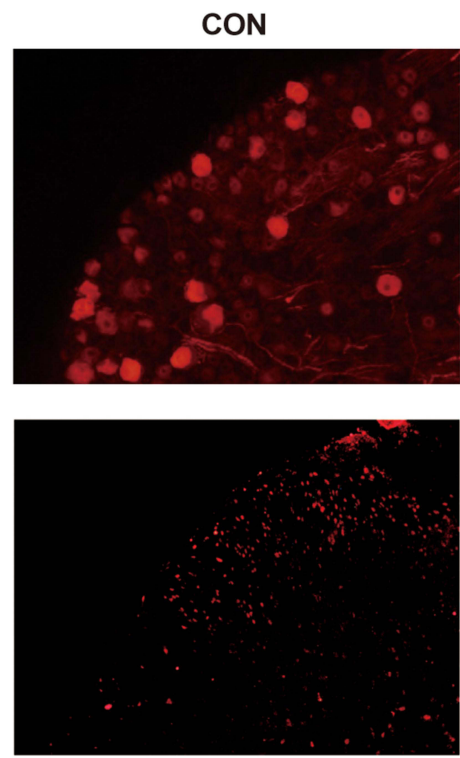

D

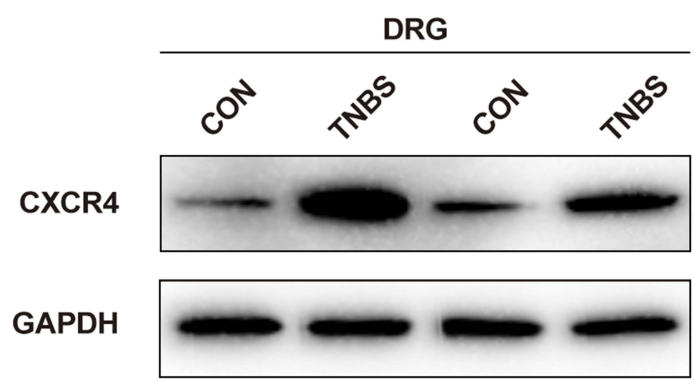

E



TNBS


F

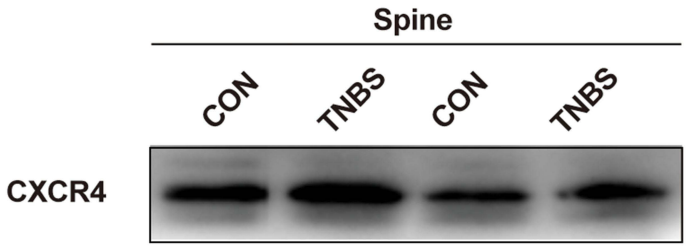

GAPDH

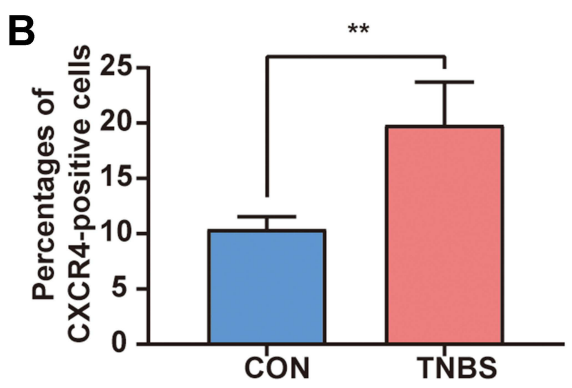

C

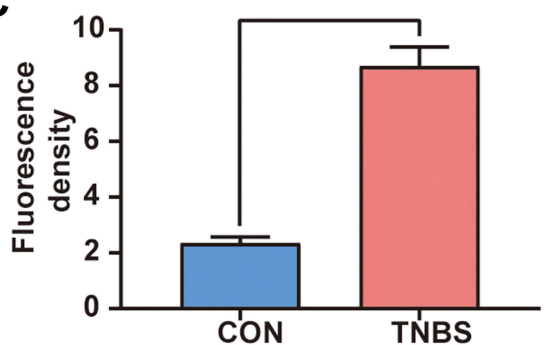

G



Figure 3 Colonic inflammation increased CXCR4 expression level in the DRG and SDH. (A) Sections of L6-SI DRG and spine were stained with anti-CXCR4 antibody. (B) Percentage of DRG neurons that were positive for CXCR4 expression $(n=5)$. (C) Quantification of the fluorescence intensity in L6-SI segments of the spine $(n=5)$. (D) CXCR4 expression levels of L6-SI DRG were detected by Western blotting. (E) Gray-scale analysis of Figure 3D $(n=6)$. $(\mathbf{F})$ Western blot analysis of CXCR4 protein in the spine, and $(\mathbf{G})$ gray-scale analysis of Western blot analysis results $(n=6)$. $* * P<0.01$.

In the SDH of the CON group, there was a low-level expression of CXCR4, which was very prominent by the 7th day after TNBS induction (Figure $3 \mathrm{~A}$ and $\mathrm{C}$ ). To further support this conclusion, Western blot analysis confirmed that experimental colitis upregulated the expression of CXCR4 in the lumbosacral spinal cord and corresponding ganglia (Figure 3D-G).

\section{Intrathecal Injection of AMD3 100 Improves Colon Inflammation-Induced Bladder Overactivity}

Owing to the expression of CXCR4 was increased in the primary afferent pathway following inflammation of the colon, we hypothesized that it may be related to colon-tobladder neural crosstalk. We detected the effect of CXCR4 
A
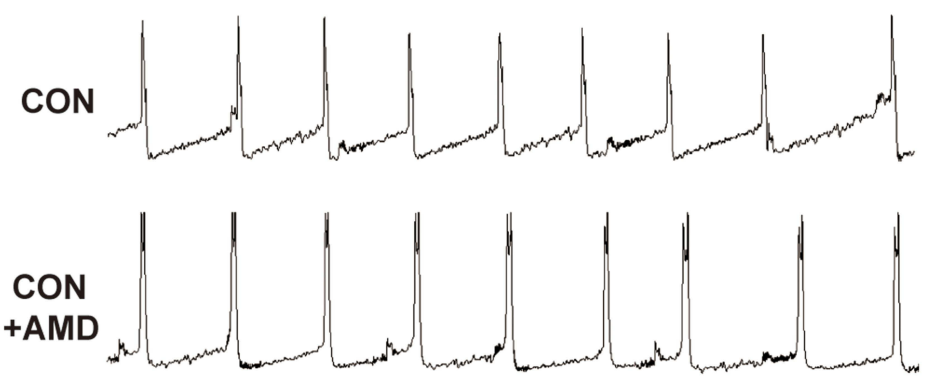



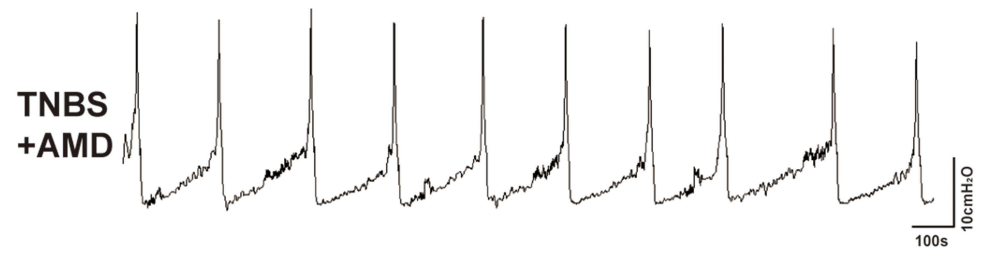

B
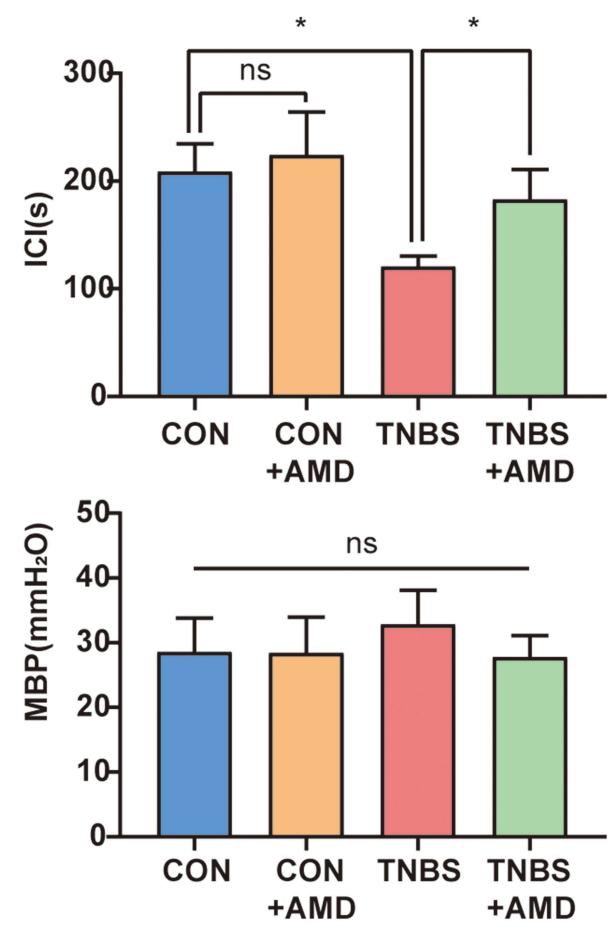

Figure 4 Intrathecal AMD3100 improves bladder voiding function in colitis rats. (A) Pressure waveform during urination of the CON group, CON+AMD group, TNBS group, and TNBS+AMD group. (B) Data analysis of the intercontraction interval $(\mathrm{ICl})(\mathrm{n}=5)$. (C) Data analysis of the maximum bladder pressure $(\mathrm{MBP})(\mathrm{n}=5)$. $* P<0.05$. Abbreviation: NS, not significant.

antagonist on bladder overactivity and hyperalgesia induced by TNBS. Cystometry was performed on conscious rats after colitis induction for 7 days. As shown in Figure 4A, the urodynamic curves from the four separate groups were recorded. Similar to previous reports, ${ }^{17}$ the TNBS group exhibited marked symptoms of bladder overactivity when compared with the CON group.

The ICI in the TNBS group was substantially shorter than that in the CON and CON+AMD groups. AMD3100 treatment significantly shortened the ICI of colitis rats (Figure 4B). Furthermore, AMD3100 can weakly decrease MBP, but differences in MBP between these four groups were less evident (Figure 4C).

\section{AMD3 100 Reversed Colon Inflammation-Induced Bladder Hyperalgesia and Mastocytosis}

Two kinds of nociceptive behaviors caused by RTX in the bladder, freezing, and licking events were counted to evaluate bladder nociception (Figure 5A and B). In comparison to the CON group, the TNBS group had more freezing and licking events. There were no obvious differences in RTX-induced nociceptive behaviors between the $\mathrm{CON}$ and $\mathrm{CON}+\mathrm{AMD}$ groups. However, intrathecal injection of AMD3100 treatment improved bladder hyperalgesia in rats induced by colon inflammation, with fewer licking and freezing events than the TNBS group. Because the activation of bladder mast cells after colitis promotes abnormal bladder function, ${ }^{22}$ we also assessed the effects of AMD3100 therapy on the number of bladder mast cells (Figure 5C). Toluidine blue staining indicates that colon inflammation caused bladder mast cells to increase by 2 3 times, which was reversed by blocking CXCR4 (Figure 5D).

\section{Inhibition of TNBS-Mediated Spinal Cord ERK Activation by AMD3100}

CXCR4 activation stimulated by ligands initiates multiple signal pathways, including the ERK pathway. ${ }^{23}$ To further investigate the possible molecular mechanisms of the antibladder-colon cross-organ sensitization effect of CXCR4, we used Western blot to check the protein level of ERK1/2 and p-ERK1/2 in the spinal cord with or without AMD3100 treatment. The phosphorylation of spinal cord ERK1/2 in the TNBS group was 3 times that of the CON group, and this change was attenuated by AMD3100 (Figure 6A and B). However, differences in p-ERK1/2 of DRG among these groups were less evident (Figure 6C 
A

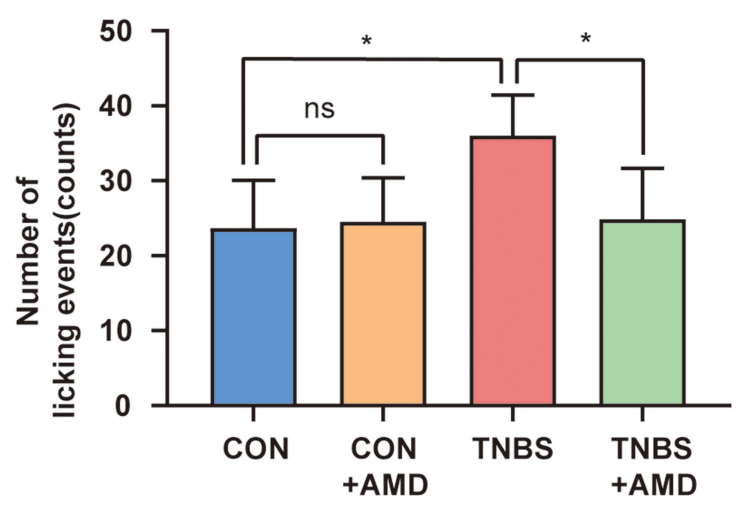

C
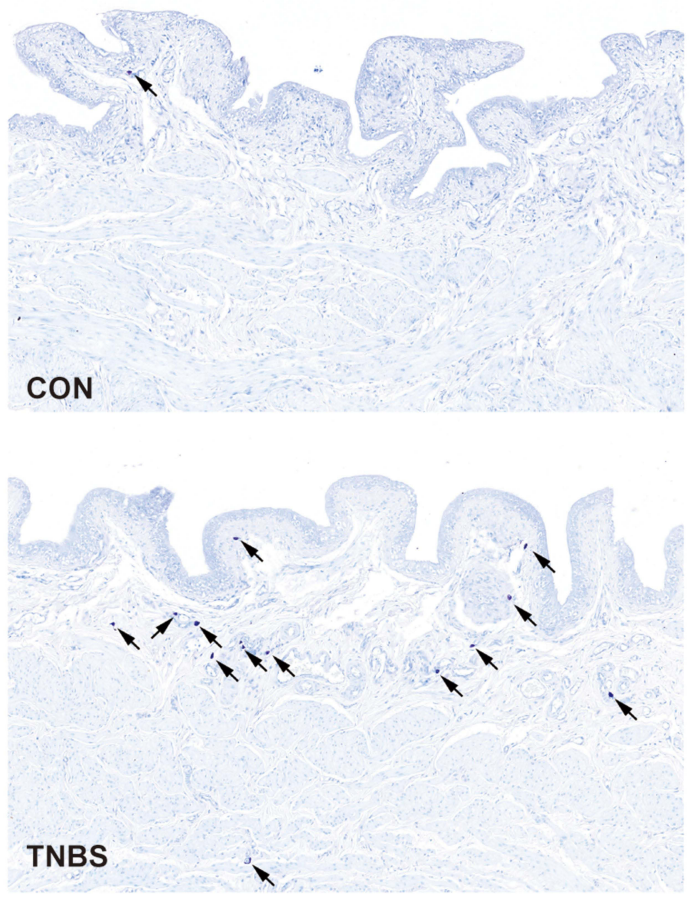

B



\section{CON+AMD}

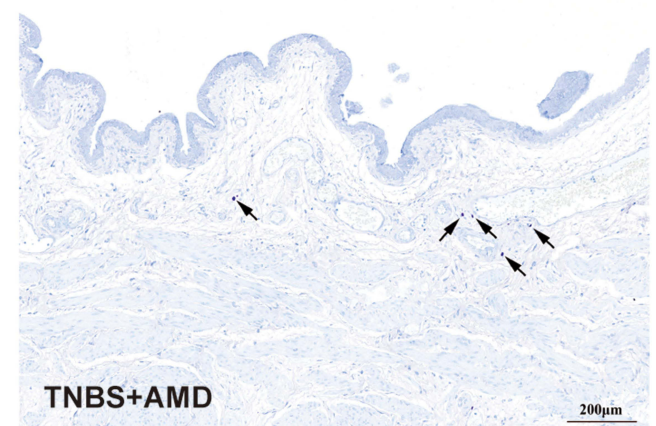

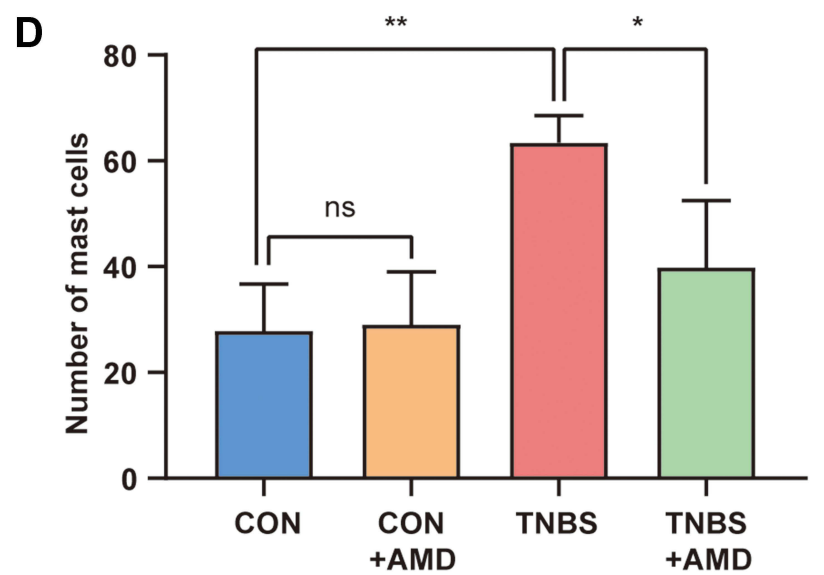

Figure 5 Intrathecal AMD3100 reduces the number of nociceptive behavior and bladder mast cells. $(\mathbf{A})$ The number of licking events caused by resiniferatoxin $(\mathrm{RTX})$ ( $\mathrm{n}=6$ ). (B) The number of RTX-induced freezing events during $15 \mathrm{~min}(\mathrm{n}=6)$. (C) Representative toluidine-blue staining images of the rat bladder. Black arrows indicate mast cells. (D) Total bladder mast cells were counted in each group $(n=5)$. *P $<0.05, * * p<0.01$. Abbreviation: NS, not significant. 
and D). To examine this further, the protein expressions of p-ERK1/2 were determined using Immunofluorescence. Seven days after colon inflammation, a significant increase in p-ERK1/2 immunostaining was observed in the dorsal horn. The intraperitoneal administration of AMD3100 inhibited the expression of p-ERK $1 / 2$ in SDH. We also found that the number of CXCR4-positive neurons in DRG showed no difference between these groups (Figure 6E), which was consistent with the Western blot data.
A

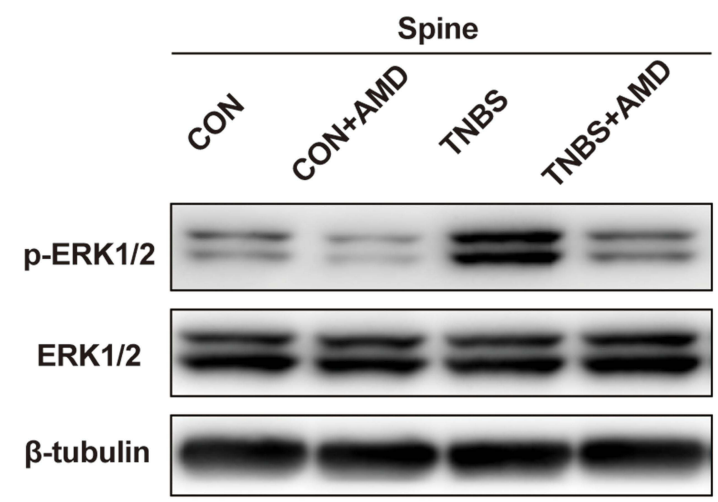

B

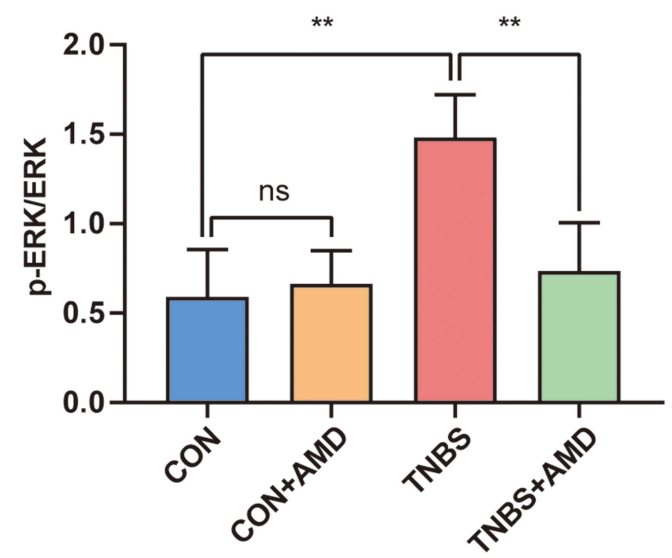

E CON
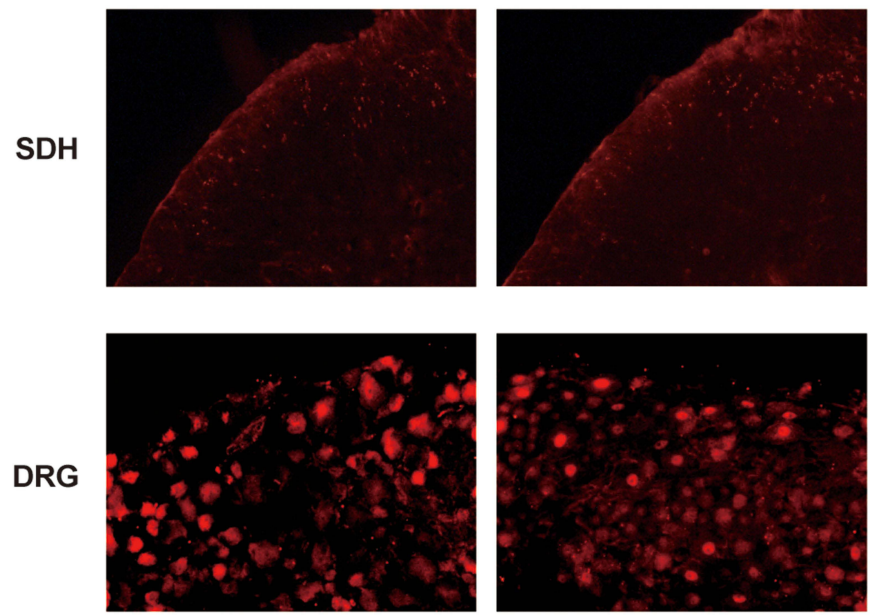

C

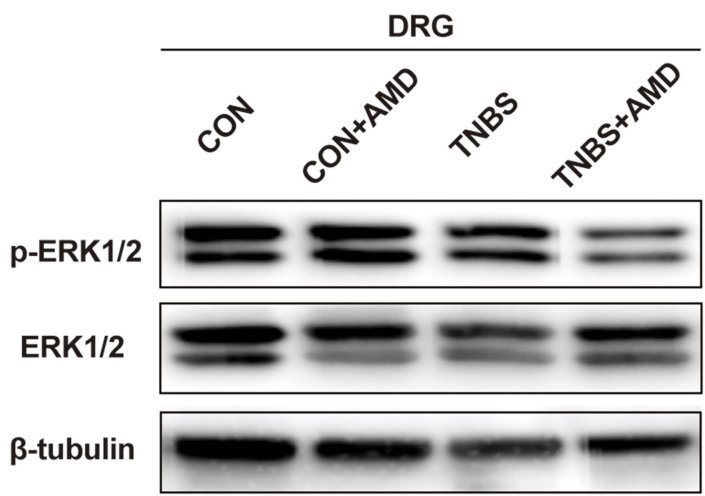

D

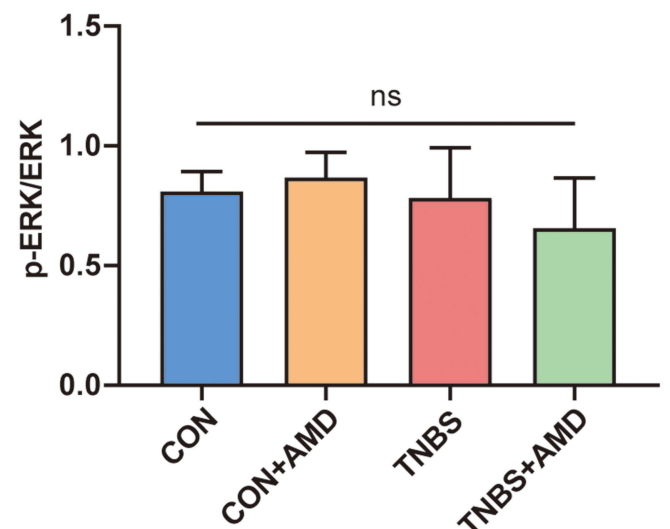

TNBS

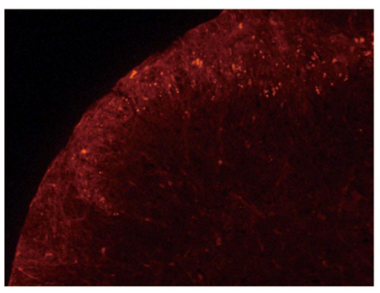

TNBS+AMD
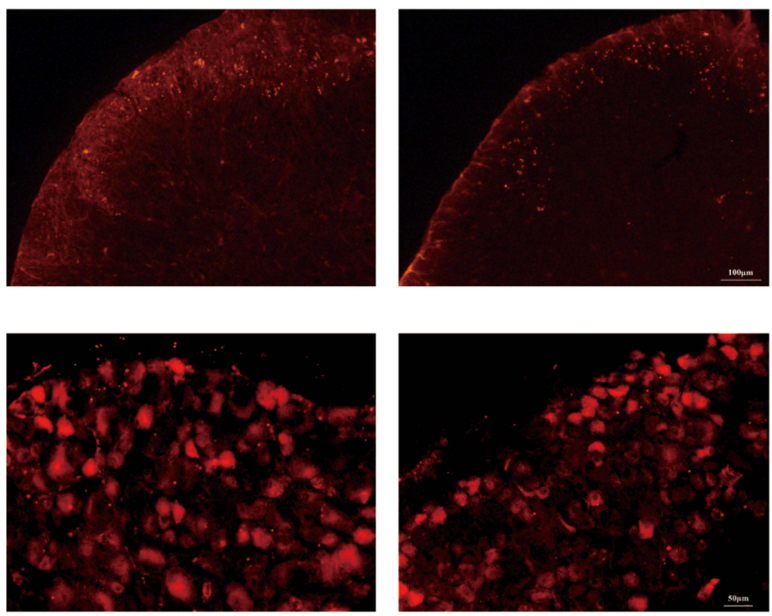

Figure 6 Intrathecal AMD3I00 decreases colitis-induced p-ERKI/2 in the spinal dorsal horn (SDH) but not DRG. (A) Representative Western blot results of p-ERKI/2 expression level and (B) quantification of Western blot analysis in SDH $(n=6)$. (C) Western blot results of $p$-ERKI/2 expression level and $(\mathbf{D})$ quantification of Western blot analysis in DRG $(n=6)$. (E) p-ERKI/2 expression in L6-SI SDH and DRG. **p $<0.01$.

Abbreviation: NS, not significant. 


\section{Attenuation of Colitis-Induced Bladder Overactivity and Hyperalgesia by Intrathecal Injection of PD98059}

To further investigate whether spinal p-ERK1/2 upregulation leads to bladder overactivity and hyperalgesia, we directly intrathecally injected PD98059, an ERK inhibitor, 7 consecutive days after the injection of TNBS. Consistent with the expected results, treatment with PD98059 resulted in downregulation of spinal p-ERK1/2 in rats with colitis, but not normal rats (Figure $7 \mathrm{~A}$ and $\mathrm{B}$ ). We then determined the effect of PD98059 on bladder hyperalgesia. The Injection of PD98059 significantly reduced the number of two nociceptive responses in TNBS rats. However, PD98059 has minimal impact on the nociceptive behavior of healthy rats (Figure $7 \mathrm{C}$ and $\mathrm{D}$ ). In addition, the TNBS+PD group showed a consistently longer mean ICI compared with the TNBS groups. MBP tended to decrease after PD98059 treatment, although the effect was not significant (Figure 7E-G).

\section{Discussion}

In this study, intracolonic administration of TNBS increased the expression of CXCR4 in the colon and bladder and their common afferent pathways. Intrathecal injection of AMD3100 reversed bladder overactivity and hyperalgesia during colonic inflammation. Further, AMD3100 significantly reduced p-ERK1/2 in the SDH but not in the DRG, and treatment with PD98059 alleviated overactive bladder and hyperalgesia caused by colitis. These results suggest that upregulation of CXCR4 in the lumbosacral spinal cord and corresponding DRG promotes bladder dysfunction induced by colitis, and that CXCR4 was partially involved in colon-bladder crosstalk via the activation of spinal cord ERK signals.

There is a bidirectional relationship between IC/BPS and gut diseases, one of which leads to a high risk of another. Central sensitization is considered to be the pathophysiological basis of bladder hypersensitivity after colitis. ${ }^{7}$ Under normal conditions, the bladder is mainly dominated by A $\delta$ fibers that are responsible for sensing the pressure and volume of the bladder. Unmyelinated $\mathrm{C}$ fibers do not participate in normal bladder function and are only activated by high-intensity noxious and chemical stimuli. ${ }^{24}$ When the colon is damaged or inflamed, injury stimulation leads to repeated activation of $\mathrm{C}$ fibers, which induces changes in the excitability and plasticity of neurons in the common afferent nerve pathway of the spinal cord and colon. The excitability threshold of these hypersensitive neurons is reduced, leading to subliminal stimulation under normal conditions that can also activate $\mathrm{A} \delta$ and $\mathrm{C}$ fibers, and further cause bladder overactivity and hyperalgesia. ${ }^{7}$

The massive activation of spinal microglia induced by colitis exacerbates central sensitization and leads to overactive bladder and hyperalgesia. ${ }^{10}$ At the level of the peripheral nervous system, colitis leads to increased expression of brain-derived neurotrophic factors, TRPV1, substance $\mathrm{P}$, and other noxious substances and receptors in DRG neurons, and these noxious substances and receptors promote the occurrence of peripheral sensitization and cause bladder functional abnormalities. ${ }^{17,25,26}$ These results indicate that central and peripheral sensitization is related to overactive bladder and hyperalgesia induced by colitis.

CXCR4 participates in inflammation of the bladder and colon, and inhibitors of CXCR4 can improve organ function and reduce inflammation. ${ }^{15,27-29}$ Inflammatory bowel disease leads to higher expression of CXCR4 in the colon, as observed in human patients, ${ }^{27,30}$ animal models, ${ }^{31}$ and in the present study. Several studies have revealed that CXCR4 affects intestinal physiology by regulating the intestinal epithelial barrier and the release of inflammatory factors. $^{28,29}$ Furthermore, CXCR4 expression rose significantly in the bladder mucosa of rats with chemical cystitis. ${ }^{15}$ The use of CXCR4 inhibitors can improve voiding dysfunction caused by bladder inflammation and obesity. $^{15,32}$

CXCR4 is an important modulator of nociception and pain sensitivity beyond its involvement in inflammation. Studies on rodents have shown that CXCR4 is expressed in DRG and spinal cord. ${ }^{12}$ CXCL12 can induce the firing frequency of nociceptive neurons in DRG of normal mice, ${ }^{33}$ and blocking CXCR4 with drugs can reverse the excessive excitement of nociceptive neurons. ${ }^{34}$ Peripheral stimulation can also activate CXCR4 in the spinal cord, and further reduce the perception threshold of noxious stimuli. $^{23}$ Genetic deletion of CXCR4 in rats supports its role in central and peripheral sensitization. Previous studies have revealed that the knockout of CXCR4 prevents mechanical allodynia induced by painful diabetic neuropathy. ${ }^{33}$ In our research, CXCR4 in L6-S1DRG and corresponding $\mathrm{SDH}$ increased significantly following 7 days of colitis. We also observed that preferential expression of CXCR4 in nociceptive small or medium DRG neurons and SDH under colitis conditions, consistent 
A

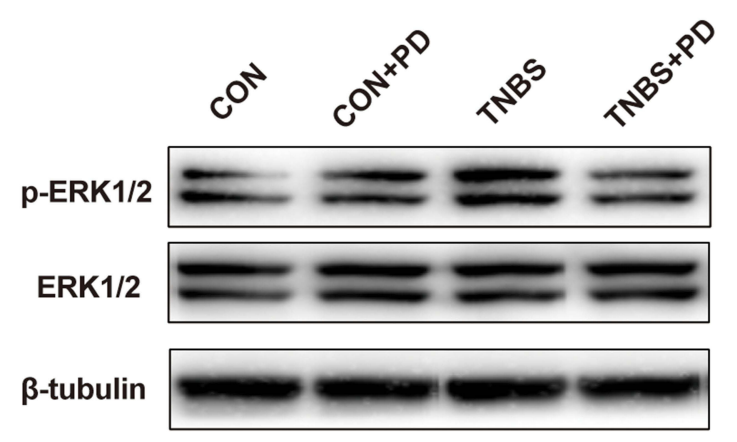

C

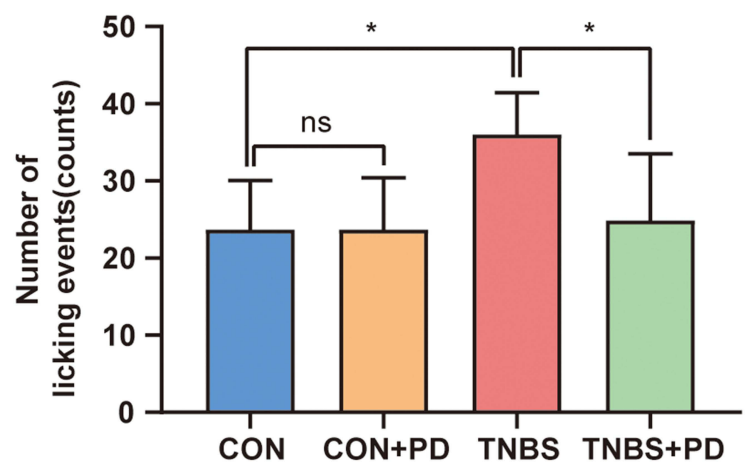

E
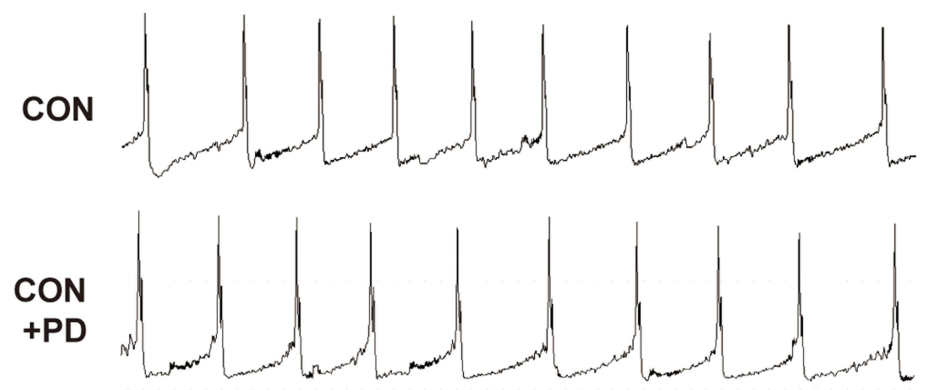

TNBS
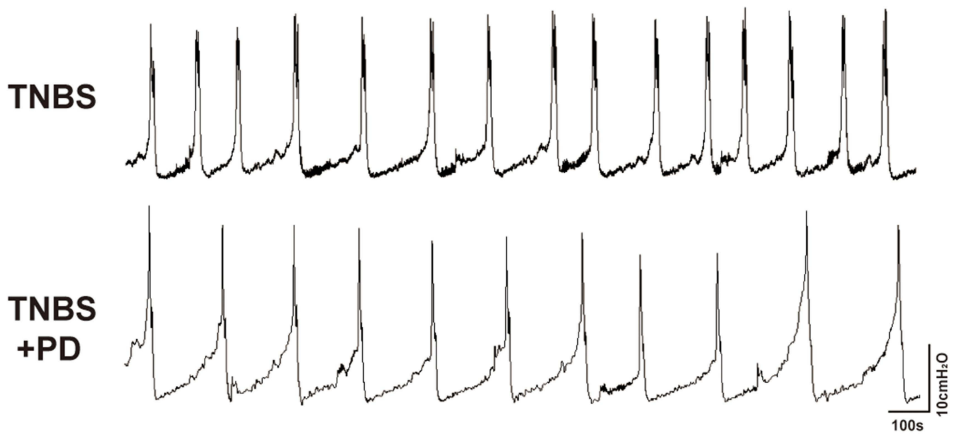

B



D



F


Figure 7 ERK antagonist decreases TNBS-induced bladder overactivity and hyperalgesia. (A) Western blot analysis of $\mathrm{p}$-ERKI/2 expression level and (B) quantification of Western blot analysis in the spinal cord $(n=6)$. The effects of PD98059 on RTX-induced nociceptive behaviors: licking events $(\mathbf{C})$ and freezing events $(\mathbf{D})$ ( $n=6)$. $(\mathbf{E})$ Pressure waveform during urination of the CON group, CON+PD group, TNBS group, and TNBS+PD group. $(\mathbf{F}-\mathbf{G})$ Statistical analysis of the ICl and MBP $(n=5)$. $* P<0.05$, $* * P<0.01$.

Abbreviation: NS, not significant. 
with that of CXCR4 in neuropathic and inflammatory pain. ${ }^{35}$ Intrathecal administration of AMD3100 significantly ameliorated TNBS colitis-induced bladder overactivity and bladder hyperalgesia but did not yield the same result in untreated rats. Therefore, the abnormal excitability of bladder afferent signals during colitis may be caused by CXCR4-mediated peripheral and central sensitization. Pharmacological intervention on CXCR4 is effective in reversing colon-bladder cross-organ sensitization and normalizing bladder function.

Noxious stimulation from the colon causes the bladder sensory neurons to be cross-activated and induces the release of neuropeptides from the axons of these neurons. $^{25}$ Neuropeptides stimulate mast cells, causing them to release harmful and pro-inflammatory substances such as neurotrophic factors, inflammatory factors, histamine, and serotonin, ${ }^{36}$ which stimulate hypersensitivity of the bladder afferent nerves adjacent to the mast cells. $^{22,37,38}$ As a key participant in central sensitization, spinal cord microglia has been reported to activate during the above process and promote the occurrence of overactive bladder and mastocytosis. ${ }^{10}$ There is direct evidence that CXCR4 is expressed in microglia and blockade of CXCR4 can inhibit the activation of spinal cord microglia in painful conditions. ${ }^{39,40}$ In this study, AMD3100 treatment reduced the number of mast cells in the bladder. Thus, bladder hypersensitivity caused by colitis is partly attributable to the increase in bladder mast cells caused by the upregulation of CXCR4 in the bladder afferent pathway.

Many previous studies have shown that the permeability of the bladder epithelium increases significantly after colon inflammation. ${ }^{41,42}$ Mast cell activation has been proved to be a key factor, and mast cell stabilizers can reverse the increase in epithelial permeability. ${ }^{22}$ The permeability change of the urothelium is an important cause of bladder pain and urination symptoms. ${ }^{43}$ The predisposing factors for increased epithelial permeability are complex, such as the damage of the epithelial barrier and the increase of paraepithelial transport. ${ }^{44}$ Interestingly, we found that the expression of CK20 in the TNBS group and the CON group was similar, which indicates that the integrity of the bladder epithelial umbrella cell layer did not change after colitis. The study by Towner et al also proved this. ${ }^{45}$ These results indicate that the neuropeptides released by mast cells are involved in regulating the permeability of the bladder epithelium through a neuromodulation mechanism, ${ }^{46}$ rather than directly destroying the epithelial barrier like acrolein and other toxins. In addition, neuropeptide-induced enhanced expression of macrophage migration inhibitory factor, a non-homologous ligand of CXCR $4,{ }^{47}$ might explain the enhanced expression of CXCR4 in the bladder epithelium. $^{48}$

The spinal cord serves as the end of a shared pathway that accepts afferents from the colon and bladder. The central terminals of colon and bladder converge and entangle in SDH. ${ }^{9}$ Previous studies have shown that ERK activation in the SDH leads to hyperfunction of the bladder in cyclophosphamide cystitis and spinal cord injury models ${ }^{49,50}$ and bladder visceral hyperalgesia after cystitis. ${ }^{51}$ In our current study, TNBS was shown to induce activation of ERK1/2 in L6-S1 $\mathrm{SDH}$. In pain models that include plantar incisions and chronic ischemia-induced pain, activation of ERK1/2 has been shown to be a critical downstream pathway for CXCR4 to central sensitization. ${ }^{23}$ In this study, selective CXCR4 blockade inhibited SDH ERK1/2 activation in response to TNBS. This indicates that the activation of CXCR4 in the colitis model promotes the phosphorylation of ERK in SDH. We found that overactive bladder and hyperalgesia after colitis were inhibited by chronic intrathecal injection of PD98059. These results indicate that the activation of CXCR4 in the spinal cord after colitis caused an increase in ERK phosphorylation, thereby inducing high excitability in the bladder afferent pathway in the spinal cord. However, the expression of p-ERK1/2 in DRG was not affected by chronic colitis and AMD3100 treatment. The same results were previously found in rat models of cystitis and colitis. ${ }^{52,53}$ The ERK in DRG may be activated in the early stage of colon inflammation and return to normal after the activation of ERK in the spinal cord. That is, harmful stimuli induce peripheral sensitization and then central sensitization.

\section{Conclusion}

In summary, current research demonstrates that upregulation of $\mathrm{CXCR} 4$ contributes to colon-bladder crossorgan sensitization. Moreover, blocking CXCR4 with AMD3100 attenuated the bladder overactivity and hyperalgesia induced by colitis via lowering the activation of spinal ERK. Therefore, our findings help elucidate the pathophysiology of cross-organ sensitization and visceral hypersensitivity induced by colitis in rats, and the CXCR4/ERK signal may be a potential therapeutic target for comorbidity between digestive and genitourinary tracts. 


\section{Acknowledgments}

This work was supported by the National Natural Science Foundation of China (grant number: 81974101) and grant from Clinical Research Talent Training Plan of Army Medical University (2018XLC3033).

\section{Disclosure}

The authors declare that they have no conflicts of interest regarding this work nor the publication of this paper.

\section{References}

1. Hanno PM, Erickson D, Moldwin R, Faraday MM, American Urological A. Diagnosis and treatment of interstitial cystitis/bladder pain syndrome: AUA guideline amendment. J Urol. 2015;193 (5):1545-1553. doi:10.1016/j.juro.2015.01.086

2. Grundy L, Brierley SM. Cross-organ sensitization between the colon and bladder: to pee or not to pee? Am J Physiol Gastrointest Liver Physiol. 2018;314(3):G301-G308. doi:10.1152/ajpgi.00272.2017

3. Kaplan SA, Dmochowski R, Cash BD, Kopp ZS, Berriman SJ, Khullar V. Systematic review of the relationship between bladder and bowel function: implications for patient management. Int J Clin Pract. 2013;67(3):205-216. doi:10.1111/ijcp.12028

4. Alagiri M, Chottiner S, Ratner V, Slade D, Hanno PM. Interstitial cystitis: unexplained associations with other chronic disease and pain syndromes. Urology. 1997;49(5ASuppl):52-57. doi:10.1016/S00904295(99)80332-X

5. Chang KM, Lee MH, Lin HH, Wu SL, Wu HC. Does irritable bowel syndrome increase the risk of interstitial cystitis/bladder pain syndrome? A cohort study of long term follow-up. Int Urogynecol $J$. 2021;32(5):1307-1312. doi:10.1007/s00192-021-04711-3

6. Brumovsky PR, Gebhart GF. Visceral organ cross-sensitization - an integrated perspective. Auton Neurosci. 2010;153(1-2):106-115. doi:10.1016/j.autneu.2009.07.006

7. Reynolds WS, Dmochowski R, Wein A, Bruehl S. Does central sensitization help explain idiopathic overactive bladder? Nat Rev Urol. 2016;13(8):481-491. doi:10.1038/nrurol.2016.95

8. Ustinova EE, Fraser MO, Pezzone MA. Colonic irritation in the rat sensitizes urinary bladder afferents to mechanical and chemical stimuli: an afferent origin of pelvic organ cross-sensitization. $\mathrm{Am}$ J Physiol Renal Physiol. 2006;290(6):F1478-1487. doi:10.1152/ ajprenal.00395.2005

9. Grundy L, Harrington AM, Castro J, et al. Chronic linaclotide treatment reduces colitis-induced neuroplasticity and reverses persistent bladder dysfunction. JCI Insight. 2018;3(19). doi:10.1172/jci. insight. 121841

10. Majima T, Funahashi Y, Kawamorita N, et al. Role of microglia in the spinal cord in colon-to-bladder neural crosstalk in a rat model of colitis. Neurourol Urodyn. 2018;37(4):1320-1328. doi:10.1002/ nau. 23484

11. Luo X, Wang X, Xia Z, Chung SK, Cheung CW. CXCL12/CXCR4 axis: an emerging neuromodulator in pathological pain. Rev Neurosci. 2016;27(1):83-92. doi:10.1515/revneuro-2015-0016

12. Reaux-le Goazigo A, Rivat C, Kitabgi P, Pohl M, Melik Parsadaniantz S. Cellular and subcellular localization of CXCL12 and CXCR4 in rat nociceptive structures: physiological relevance. Eur $\quad J \quad$ Neurosci. 2012;36(5):2619-2631. doi:10.1111/j.14609568.2012.08179.x

13. Yang F, Sun W, Luo WJ, et al. SDF1-CXCR4 signaling contributes to the transition from acute to chronic pain state. Mol Neurobiol. 2017;54(4):2763-2775. doi:10.1007/s12035-016-9875-5
14. Kouzoukas DE, Meyer-Siegler KL, Ma F, Westlund KN, Hunt DE, Vera PL. Macrophage migration inhibitory factor mediates PAR-induced bladder pain. PLoS One. 2015;10(5):e0127628. doi:10.1371/journal.pone. 0127628

15. Arms L, Girard BM, Vizzard MA. Expression and function of CXCL12/CXCR4 in rat urinary bladder with cyclophosphamide-induced cystitis. Am J Physiol Renal Physiol. 2010;298(3):F589-600. doi:10.1152/ajprenal.00628.2009

16. Foster R, Jung J, Farooq A, et al. Sciatic nerve injury induces functional pro-nociceptive chemokine receptors in bladder-associated primary afferent neurons in the rat. Neuroscience. 2011;183:230-237. doi:10.1016/j.neuroscience.20 11.03 .035

17. Xia CM, Gulick MA, Yu SJ, et al. Up-regulation of brain-derived neurotrophic factor in primary afferent pathway regulates colon-tobladder cross-sensitization in rat. J Neuroinflammation. 2012;9:30. doi:10.1186/1742-2094-9-30

18. Yang Y, Zhang H, Lu Q, et al. Suppression of adenosine A2a receptors alleviates bladder overactivity and hyperalgesia in cyclophosphamide-induced cystitis by inhibiting TRPV1. Biochem Pharmacol. 2021;183:114340. doi:10.1016/j.bcp.2020.114340

19. Luo J, Yang C, Luo X, et al. Chlorogenic acid attenuates cyclophosphamide-induced rat interstitial cystitis. Life Sci. 2020;254:117590. doi:10.1016/j.1fs.2020.117590

20. Kawamorita N, Yoshikawa S, Kashyap M, et al. Liposome based intravesical therapy targeting nerve growth factor ameliorates bladder hypersensitivity in rats with experimental colitis. J Urol. 2016;195 (6):1920-1926. doi:10.1016/j.juro.2015.12.090

21. Romih R, Korosec P, de Mello W Jr, Jezernik K. Differentiation of epithelial cells in the urinary tract. Cell Tissue Res. 2005;320 (2):259-268. doi:10.1007/s00441-004-1005-4

22. Fitzgerald JJ, Ustinova E, Koronowski KB, de Groat WC, Pezzone MA. Evidence for the role of mast cells in colon-bladder cross organ sensitization. Auton Neurosci. 2013;173(1-2):6-13. doi:10.1016/j.autneu.2012.09.002

23. Hu Q, Zheng X, Li X, et al. Electroacupuncture alleviates mechanical allodynia in a rat model of complex regional pain syndrome Type-I via suppressing spinal CXCL12/CXCR4 signaling. $J$ Pain. 2020;21(9-10):1060-1074. doi:10.1016/j.jpain.2020.01.007

24. Birder L, de Groat W, Mills I, Morrison J, Thor K, Drake M. Neural control of the lower urinary tract: peripheral and spinal mechanisms. Neurourol Urodyn. 2010;29(1):128-139. doi:10.1002/nau.20837

25. Pan XQ, Gonzalez JA, Chang S, Chacko S, Wein AJ, Malykhina AP. Experimental colitis triggers the release of substance $\mathrm{P}$ and calcitonin gene-related peptide in the urinary bladder via TRPV1 signaling pathways. Exp Neurol. 2010;225(2):262-273. doi:10.1016/j. expneurol.2010.05.012

26. Lei Q, Malykhina AP. Colonic inflammation up-regulates voltage-gated sodium channels in bladder sensory neurons via activation of peripheral transient potential vanilloid 1 receptors. Neurogastroenterol Motil. 2012;24(6):575-585, e257. doi:10.1111/ j.1365-2982.2012.01910.x

27. Werner L, Guzner-Gur H, Dotan I. Involvement of CXCR4/CXCR7/ CXCL12 interactions in inflammatory bowel disease. Theranostics. 2013;3(1):40-46. doi:10.7150/thno.5135

28. Mikami S, Nakase H, Yamamoto S, et al. Blockade of CXCL12/ CXCR4 axis ameliorates murine experimental colitis. $J$ Pharmacol Exp Ther. 2008;327(2):383-392. doi:10.1124/jpet.108.141085

29. Xia XM, Wang FY, Zhou J, Hu KF, Li SW, Zou BB. CXCR4 antagonist AMD3100 modulates claudin expression and intestinal barrier function in experimental colitis. PLoS One. 2011;6(11): e27282. doi:10.1371/journal.pone.0027282

30. Dotan I, Werner L, Vigodman S, et al. CXCL12 is a constitutive and inflammatory chemokine in the intestinal immune system. Inflamm Bowel Dis. 2010;16(4):583-592. doi:10.1002/ibd.21106 
31. Lin X, Wang H, Li Y, et al. Functional characterization of CXCR4 in mediating the expression of protein $\mathrm{C}$ system in experimental ulcerative colitis. Am J Transl Res. 2017;9(11):4821-4835.

32. Macoska JA, Wang Z, Virta J, Zacharias N, Bjorling DE. Inhibition of the CXCL12/CXCR4 axis prevents periurethral collagen accumulation and lower urinary tract dysfunction in vivo. Prostate. 2019;79 (7):757-767. doi:10.1002/pros.23781

33. Jayaraj ND, Bhattacharyya BJ, Belmadani AA, et al. Reducing CXCR4-mediated nociceptor hyperexcitability reverses painful diabetic neuropathy. J Clin Invest. 2018;128(6):2205-2225. doi:10.1172/ JCI92117

34. Yang F, Sun W, Yang Y, et al. SDF1-CXCR4 signaling contributes to persistent pain and hypersensitivity via regulating excitability of primary nociceptive neurons: involvement of ERK-dependent Nav1.8 up-regulation. $J$ Neuroinflammation. 2015;12:219. doi:10.1186/s12974-015-0441-2

35. Bai L, Wang X, Li Z, et al. Upregulation of chemokine CXCL12 in the dorsal root ganglia and spinal cord contributes to the development and maintenance of neuropathic pain following spared nerve injury in rats. Neurosci Bull. 2016;32(1):27-40. doi:10.1007/s12264-0150007-4

36. Kulka M, Sheen CH, Tancowny BP, Grammer LC, Schleimer RP. Neuropeptides activate human mast cell degranulation and chemokine production. Immunology. 2008;123(3):398-410. doi:10.1111/ j.1365-2567.2007.02705.x

37. Ustinova EE, Gutkin DW, Pezzone MA. Sensitization of pelvic nerve afferents and mast cell infiltration in the urinary bladder following chronic colonic irritation is mediated by neuropeptides. Am J Physiol Renal Physiol. 2007;292(1):F123-130. doi:10.1152/ajprenal.00 162.2006

38. Ustinova EE, Fraser MO, Pezzone MA. Cross-talk and sensitization of bladder afferent nerves. Neurourol Urodyn. 2010;29(1):77-81. doi:10.1002/nau.20817

39. Liu ZY, Song ZW, Guo SW, et al. CXCL12/CXCR4 signaling contributes to neuropathic pain via central sensitization mechanisms in a rat spinal nerve ligation model. CNS Neurosci Ther. 2019;25 (9):922-936. doi:10.1111/cns.13128

40. Luo X, Tai WL, Sun L, et al. Crosstalk between astrocytic CXCL12 and microglial CXCR4 contributes to the development of neuropathic pain. Mol Pain. 2016;12. doi:10.1177/1744806916636385

41. Greenwood-van Meerveld B, Mohammadi E, Tyler K, et al. Mechanisms of visceral organ crosstalk: importance of alterations in permeability in rodent models. J Urol. 2015;194(3):804-811. doi:10.1016/j.juro.2015.02.2944

42. Towner RA, Smith N, Saunders D, et al. Assessment of colon and bladder crosstalk in an experimental colitis model using contrast-enhanced magnetic resonance imaging. Neurogastroenterol Motil. 2015;27(11):1571-1579. doi:10.1111/nmo.12654
43. Hurst RE, Greenwood-van Meerveld B, Wisniewski AB, et al. Increased bladder permeability in interstitial cystitis/painful bladder syndrome. Transl Androl Urol. 2015;4(5):563-571. doi:10.3978/j. issn.2223-4683.2015.10.03

44. Montalbetti N, Rued AC, Clayton DR, et al. Increased urothelial paracellular transport promotes cystitis. Am J Physiol Renal Physiol. 2015;309(12):F1070-1081. doi:10.1152/ajprenal.002 00.2015

45. Towner RA, Smith N, Saunders D, et al. Contrast enhanced magnetic resonance imaging as a diagnostic tool to assess bladder permeability and associated colon cross talk: preclinical studies in a rat model. J Urol. 2015;193(4):1394-1400. doi:10.1016/j.juro.2014.10.120

46. Sant GR, Kempuraj D, Marchand JE, Theoharides TC. The mast cell in interstitial cystitis: role in pathophysiology and pathogenesis. Urology. 2007;69(4 Suppl):34-40. doi:10.1016/j.urology.200 6.08.1109

47. Vera PL, Iczkowski KA, Wang X, Meyer-Siegler KL. Cyclophosphamide-induced cystitis increases bladder CXCR4 expression and CXCR4-macrophage migration inhibitory factor association. PLoS One. 2008;3(12):e3898. doi:10.1371/journal. pone. 0003898

48. Meyer-Siegler KL, Vera PL. Substance P induced release of macrophage migration inhibitory factor from rat bladder epithelium. J Urol. 2004;171(4):1698-1703. doi:10.1097/01.ju.0000115883.49365.1a

49. Cruz CD, Avelino A, McMahon SB, Cruz F. Increased spinal cord phosphorylation of extracellular signal-regulated kinases mediates micturition overactivity in rats with chronic bladder inflammation. Eur J Neurosci. 2005;21(3):773-781. doi:10.1111/j.1460-9568.20 05.03893.x

50. Cruz CD, McMahon SB, Cruz F. Spinal ERK activation contributes to the regulation of bladder function in spinal cord injured rats. Exp Neurol. 2006;200(1):66-73. doi:10.1016/j.expneurol.2006.01.016

51. Lai HH, Qiu CS, Crock LW, Morales MEP, Ness TJ, Gereau R. Activation of spinal extracellular signal-regulated kinases (ERK) 1/ 2 is associated with the development of visceral hyperalgesia of the bladder. Pain. 2011;152(9):2117-2124. doi:10.1016/j.pain.201 1.05 .017

52. Qiao LY, Gulick MA. Region-specific changes in the phosphorylation of ERK1/2 and ERK5 in rat micturition pathways following cyclophosphamide-induced cystitis. Am J Physiol Regul Integr Comp Physiol. 2007;292(3):R1368-1375. doi:10.1152/ajpregu.00 570.2006

53. Yu SJ, Grider JR, Gulick MA, Xia CM, Shen S, Qiao LY. Upregulation of brain-derived neurotrophic factor is regulated by extracellular signal-regulated protein kinase 5 and by nerve growth factor retrograde signaling in colonic afferent neurons in colitis. Exp Neurol. 2012;238(2):209-217. doi:10.1016/j.expneurol.2012.08.007

\section{Publish your work in this journal}

Drug Design, Development and Therapy is an international, peerreviewed open-access journal that spans the spectrum of drug design and development through to clinical applications. Clinical outcomes, patient safety, and programs for the development and effective, safe, and sustained use of medicines are a feature of the journal, which has also been accepted for indexing on PubMed Central. The manuscript management system is completely online and includes a very quick and fair peer-review system, which is all easy to use. Visit http://www. dovepress.com/testimonials.php to read real quotes from published authors. 